zifischen Lebensmitteln waren Fisch/ Muscheln (26\%), Erdnüsse (20\%) und andere Nüsse (20\%) führend. Weitere Auslöser waren Früchte/Gemüse, Kuhmilch, Hühnereier, Weizen, Fleisch/Geflügel und Soja.

$41 \%$ der Reaktionen wurden als mittelschwer und 7\% als schwer eingestuft. In 29 Fällen wurden die Kinder stationär eingewiesen, in neun Fällen auf die Intensivstation. Patienten, die zwei Dosen Adrena- lin erhalten hatten, kamen häufiger ins Krankenhaus als Patienten mit nur einer Adrenalininjektion $(\mathrm{p}<0,001)$. Wenn allerdings die Adrenalingaben schon vor der Ankunft in der Ambulanz erfolgt waren, wurden die Kinder seltener stationär aufgenommen $(p=0,05)$. Häufiger als mit Adrenalin - der Therapie der ersten Wahl bei Anaphylaxie - waren die Kinder mit Antihistaminika und Kortikosteroiden behandelt worden (79\% vs. $92 \%$ und $89 \%$ ).
Fazit: Lebensmittel dominieren als Auslöser von anaphylaktischen Reaktionen im Kindesalter. Durch eine frühzeitige Adrenalingabe können möglicherweise stationäre Behandlungen vermieden werden.

Dr. Beate Schumacher

Huang $F$ et al. Anaphylaxis in a New York City pediatric emergency department: triggers, treatments and outcomes. J Allergy Clin Immunol 2012; 129: 162-8

\title{
Schwere Nebenwirkungen von Medikamenten: Risiko steigt mit dem Alter
}

\section{Betagte Patienten, die mehrere Medikamente einnehmen, haben ein besonders hohes Nebenwirkungsrisiko. Eine US-amerikanische Studie förderte nun zu Tage, welche Komplikationen bei welchen Patienten am häufigsten auftreten.}

\begin{abstract}
edes Jahr ereignen sich in den USA 270.000 Medikamenten-Notfälle, etwa 100.000 davon so schwer, dass eine Krankenhausaufnahme erforderlich wird. Eine von 67 notfallmäßigen Krankenhausaufnahmen erfolgt wegen Medikamentennebenwirkungen. Ein nationales Projekt soll die Anzahl vermeidbarer Krankenhauseinweisungen bis $2013 \mathrm{um} \mathrm{20 \%} \mathrm{zu}$ reduzieren. Im Rahmen des Projekts untersuchten amerikanische Forscher den Zusammenhang zwischen Alter und dem Risiko, eine schwere Medikamentennebenwirkung zu erleiden.

Demnach steigt das Nebenwirkungsrisiko mit dem Alter und mit der Zahl der eingenommenen Medikamente. Am häufigsten waren Blutungskomplikationen unter oraler Antikoagulation. Damit war Warfarin (vergleichbar mit unserem Phenprocoumon) für die alten Patienten das gefährlichste Medikament (verantwortlich für 33,3\% der Krankenhauseinweisungen), gefolgt von Insulinen (13,9\%), Thrombozytenaggregationshemmern (13,3\%) und oralen Antidiabetika (10,7\%).
\end{abstract}

Budnitz DS et al. Emergency hospitalizations for adverse drug events in older Americans. N Engl J Med 2011; 365: 2002-12

Kommentar: Krankenhauseinweisungen wegen Medikamentennebenwirkungen zählen bei älteren Menschen - nicht nur in den USA, sondern auch bei uns - zu den häufigsten Gründen für eine Krankenhauseinweisung. Von den über 65-Jährigen nehmen $40 \%$ fünf bis neun und $18 \%$ sogar zehn und mehr Medikamente ein. Wegen altersbedingter physiologischer Veränderungen und einer verminderten Widerstandskraft gegen externe Stressoren wie zum Beispiel Medikamente haben ältere Menschen ein über siebenfach höheres Risiko, eine Medikamentennebenwirkung zu erleiden, die eine notfallmäßige Krankenhauseinweisung erforderlich macht.

Je älter der Patient, desto kritischer wird das Verhältnis zwischen erwünschten und unerwünschten Medikamentenwirkungen.

Die leitliniengerechte Therapie eines 65-Jährigen ist nicht auf den gebrechlichen 85-Jährigen übertragbar. Die Indikation zur oralen Antikoagulation sollte beispielsweise beim gebrechlichen Hochbetagten besonders kritisch und erst nach sorgfältigem geriatrischen Assessment gestellt werden.

Eine weitere wichtige Erkenntnis aus der Studie: Negativlisten mit Medikamenten, die für alte Menschen prinzipiell ungeeignet sind, helfen uns kaum weiter. Nur ein kleiner Teil der Nebenwirkungen wurde von solchen negativ gelisteten Arzneien verursacht. Die Hauptgefahr geht von Medikamenten aus, die aus der Altersmedizin nicht wegzudenken sind. Ihr sorgfältig abgewogener Einsatz - nicht alles für jeden - ist der Schlüssel zum Erfolg. Das geriatrische Assessment hilft wesentlich bei der Entscheidung „Wen sollte ich behandeln und wen besser nicht?"

Dr. Joachim Zeeh Geriatrische Fachklinik Georgenhaus Meiningen

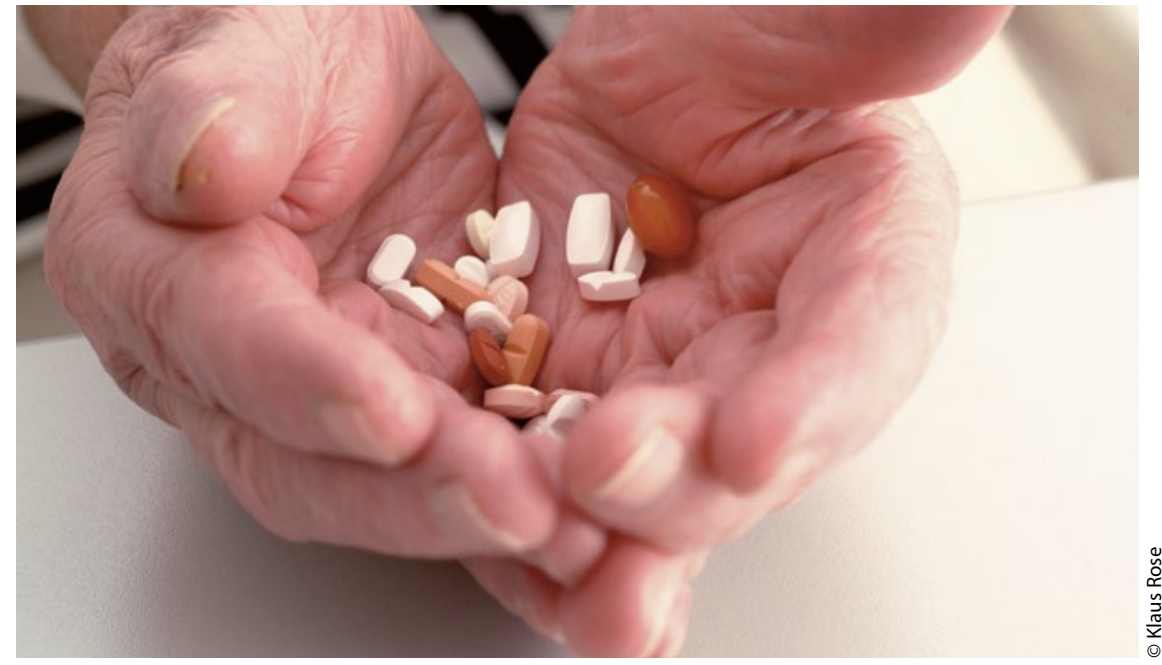

Hohes Nebenwirkungsrisiko: Multimedikation bei älteren Patienten 